\title{
El papel de la gestión cooperativa en la mejora del funcionamiento técnico- económico de explotaciones ovinas
}

\author{
Gaspar, P. ${ }^{1,4 @ ; ~ E s c r i b a n o, ~ M .2,4 ; ~ P u l i d o, ~ F . ~}{ }^{3,4}$; Rodríguez-Ledesma, A. ${ }^{1,4}$; Mesías, F.J. ${ }^{3,4}$ y Pulido, A.F. 3,4
}

\begin{abstract}
'Departamento de Producción Animal y Ciencia de los Alimentos. Escuela de Ingenierías. Universidad de Extremadura. Badajoz. España. ${ }^{2}$ Departamento de Producción Animal y Ciencia de los Alimentos. Facultad de Veterinaria. Universidad de Extremadura. Cáceres. España. ${ }^{3}$ Departamento de Economía. Escuela de Ingenierías Agrarias. Universidad de Extremadura. Badajoz. España. ${ }^{4}$ Instituto de Investigación de Recursos Agrarios (INURA). Badajoz. España.
\end{abstract}

PALABRAS CLAVE ADICIONALES

Indicadores productivos.

Ovino de carne.

España.

Análisis técnico-económico

\section{RESUMEN}

Las explotaciones ovinas en el SO de España se han visto sometidas en los últimos años a distintas presiones, tanto comerciales por los cambios de consumo como políticas por las diversas reformas de la Política Agraria Comunitaria, que se han traducido en procesos de abandono de la actividad o de intensificación para aquellos ganaderos que se han mantenido en el sector. Estos sistemas, no obstante, son enormemente frágiles, además de tener un papel fundamental en la provisión de servicios ambientales. En este contexto se ha estudiado una muestra de 101 explotaciones ovinas pertenecientes a cooperativas y localizadas en el SO de España analizando indicadores de gestión técnica y económica. Se observa que la integración de las explotaciones en estructuras cooperativas puede mejorar las explotaciones y asegurar la persistencia de las mismas gracias a la provisión de servicios adicionales al ganadero, a la generación de valor añadido y a la estabilidad en los precios percibidos.

\section{The role of cooperative management in improving the technical and economic performance of sheep farms}

\section{SUMMARY}

Sheep farms in SW Spain have been exposed to various pressures derived from changes in the consumption patterns and policy changes linked to several reforms of the Common Agricultural Policy in the last years, which has translated into the abandonment of the farm activity or the intensification process for those farms which have continued in the sector. These systems, however, are extremely fragile, as well as they have a key role in providing environmental services. In this context, we have analyzed a sample of 101 sheep farms belonging to cooperatives and located in Spain SW. Technical and economic indicators related to farm management have been studied. It is noted that the integration of farms into cooperatives can improve farm structure and ensure their persistence, due to the provision of additional services to the farmer, the generation of an added value and an increased price stability.

\section{INFORMACIÓN}

Cronología del artículo.

Recibido/Received: 01.03.2016

Aceptado/Accepted: 08.06.2016

On-line: 15.09 .2016

Correspondencia a los autores/Contact e-mail:

pgaspar@unex.es

\section{INTRODUCCIÓN}

El ovino en España se cría principalmente en sistemas extensivos o semi-extensivos asociado a la explotación de razas autóctonas debido a su buena adaptación a las condiciones ambientales adversas. Es frecuente el aprovechamiento por el ovino de las tierras de pastoreo, así como de diferentes cultivos forrajeros y de cereales de secano.

En el centro oeste peninsular, la producción ovina está basada fundamentalmente en un sistema de explotación extensivo, generalmente asociado a zonas poco aprovechadas por el ganado porcino y bovino, siendo frecuente su ubicación en suelos pobres y con escasa o nula presencia de arbolado, como las dehesas poco arboladas y los pastizales de secano. Estas explotaciones en su mayoría se encuentran especializadas en la producción cárnica.

El peso del ovino en Extremadura es significativo, con un censo de 3,4 millones de cabezas, lo que representa el 20,7\% del censo a nivel nacional (MAGRAMA, 2014). Las sucesivas reformas de la PAC y los continuos cambios en el mercado han conducido a una disminución de la rentabilidad de las explotaciones. Como consecuencia, se han producido cambios en el uso del suelo, que se han podido materializar en una intensi- 
ficación productiva y en el abandono de explotaciones ovinas (Gaspar et al., 2008; Gaspar et al., 2009; RipollBosch et al., 2012).

En las últimas décadas la reducción en cuanto al número de granjas y de animales ha sido considerable. El abandono de esta actividad ovina ha sido objeto de preocupación por las distintas instituciones tanto a nivel europeo, como nacional o regional. En concreto, en España (segundo productor de carne de ovino en UE-28) cabe destacar que desde 2002 la producción de carne de cordero se ha reducido aproximadamente un 50\% (MAGRAMA, 2014).

En este sentido, existe un cierto consenso sobre la fragilidad de los sistemas de ovino de carne ubicados en regiones mediterráneas (De Rancourt et al., 2006), a pesar del papel crucial que juegan en el mantenimiento medioambiental y socioeconómico de áreas desfavorecidas de Europa (Bernués et al., 2011). Un ejemplo de este importante papel es la contribución del ovino en pastoreo en la lucha contra el cambio climático a través de la protección contra los incendios forestales (Mena et al., 2016).

De manera paralela el sector del ovino de carne está sufriendo una transformación, tanto en la gestión de sus sistemas productivos como en su comercialización, para conseguir una estabilidad de los precios a lo largo del año y el desarrollo de nuevas presentaciones y/o formatos del producto que lo hagan más atractivo para el consumidor. El objetivo es que los productores puedan obtener mayores rendimientos económicos mediante la mejora de la gestión de sus explotaciones y de la comercialización de sus productos. Ambos niveles pueden ser abordados desde la integración de las explotaciones en un sistema cooperativo que pueda hacer frente a la actual situación y mejorar su competitividad (Ruiz et al., 2014), suponiendo además esta integración uno de los aspectos claves para el mantenimiento y viabilidad de los sistemas extensivos (Horrillo et al., 2016).

Tal es así que actualmente el sector del ovino de carne se ha visto envuelto en los últimos años en un proceso de integración cooperativa muy importante. Este fenómeno de concentración cooperativa ha afectado intensamente al sector ovino extremeño y ha llevado a aglutinar en la actualidad el 64,7 \% de los 2,3 millones de ovejas de carne en estructuras cooperativas y SATs, lo que ha llevado a un incremento de su rentabilidad (Pozo y Agudo, 2013).

En este contexto, es de singular importancia conocer las características tanto técnicas como económicas de las explotaciones ovinas integradas en cooperativas. Para ello se va a realizar un análisis comparado de tres de las principales cooperativas socias de la Cooperativa de segundo grado OVISO, en las que se estudiarán los principales parámetros de gestión mediante el análisis de 101 explotaciones. El trabajo pretende avanzar en el conocimiento de los factores y características específicas, tanto a nivel técnico como económico, de dichas explotaciones y se justifica por la insuficiente investigación en este ámbito.

El análisis se aborda a través del estudio de diferentes indicadores técnicos y económicos elaborados a partir de la bibliografía existente (Gibon et al., 1999; Escribano et al., 2001; Milán et al., 2003; Gaspar et al., 2008 y 2009; Riedel et al., 2005; Usai et al., 2006), así como de la experiencia en trabajos anteriores de los autores.

\section{MATERIAL Y METODOS}

\section{ÁREA DE ESTUDIO}

Los datos utilizados en este estudio corresponden al análisis de 101 explotaciones de ovino de tres de las cooperativas más significativas pertenecientes al

Tabla I. Características básicas de las explotaciones analizadas (Basic characteristics of the analyzed farms).

\begin{tabular}{|c|c|c|c|c|c|}
\hline & & Coop 1 & Coop 2 & Coop 3 & Total \\
\hline Número explotaciones & & 33 & 26 & 48 & 101 \\
\hline Superficie total (ha) & & 360,39 & 326,56 & 420,83 & 379,28 \\
\hline Superficie en propiedad (ha) & & 73,09 & 274,39 & 219,16 & 187,53 \\
\hline Superficie en arrendamiento (ha) & & 287,30 & 52,17 & 201,66 & 191,75 \\
\hline Superficie de cultivo (ha) & & 52,45 & 63,93 & 112,13 & 82,01 \\
\hline Número de ovejas & & 779,76 & 1077,33 & 858,10 & 887,3 \\
\hline Número de carneros & & 25,24 & 43,27 & 30,44 & 31,95 \\
\hline Dedicación del ganadero al ovino (\%) & Sí & 6,1 & 38,5 & 91,7 & 52,3 \\
\hline \multirow[t]{3}{*}{ Raza predominante (\%) } & Merina & 50 & 57,7 & 6,3 & 31 \\
\hline & Cruzada & 38,5 & 38,5 & 91,6 & 64 \\
\hline & Cárnica & 11,5 & 3,8 & 2,1 & 5 \\
\hline $\begin{array}{l}\text { Utilización tratamientos hormonales } \\
(\%)\end{array}$ & Sí & 60,6 & 60 & 62,5 & 61,3 \\
\hline $\begin{array}{l}\text { Manejo de cubriciones: Efecto macho } \\
\text { y monta controlada (\%) }\end{array}$ & Sí & 84,8 & 46,2 & 72,9 & 70,1 \\
\hline $\begin{array}{l}\text { Separación según tipo de parto } \\
\text { (dobles y triples) (\%) }\end{array}$ & Sí & 6,1 & 96 & 8,3 & 28,3 \\
\hline
\end{tabular}


Tabla II. Índices técnicos. Media, error estándar y nivel de significación (ANOVA) en las explotaciones en función de la cooperativa de pertenencia (Technical indexes. Mean, standard error and significance level (ANOVA) in the farms according to cooperative membership).

\begin{tabular}{|c|c|c|c|c|c|c|}
\hline Indicadores & Coop 1 & Coop 2 & Coop 3 & Total & ESM & Sig. \\
\hline Carga ganadera (UGM/ha) & $0,48^{\mathrm{ab}}$ & $0,49^{a}$ & $0,39^{b}$ & 0,47 & 0,02 & ** \\
\hline Tasa de reposición reproductoras (\% ) & $15,44^{\mathrm{ab}}$ & $19,63^{a}$ & $14,42^{\mathrm{b}}$ & 16,02 & 0,75 & * \\
\hline Desvieje reproductoras (\% ) & $7,71^{\mathrm{a}}$ & $10,99^{b}$ & $9,55^{\mathrm{ab}}$ & 9,27 & 0,47 & * \\
\hline Total de corderos nacidos por oveja y año & 1,07 & 1,22 & 1,09 & 1,12 & 0,03 & NS \\
\hline Ratio ovejas reproductoras/semental & $33,62^{a}$ & $26,33^{b}$ & $29,77^{\mathrm{ab}}$ & 30,12 & 0,83 & ** \\
\hline
\end{tabular}

${ }^{*} p<0,05 y{ }^{* *} p<0,01$; ESM: Error estándar de muestreo. Diferentes índices en la misma fila señalan diferencias significativas; NS: No significativo.

grupo OVISO. El grupo OVISO es una cooperativa de segundo grado con sede en Villanueva de la Serena (Badajoz) e integrada en la actualidad en OVISUR, en lo que constituye el mayor grupo cooperativo de ovino de carne a nivel europeo. OVISO engloba a 17 cooperativas cuyos socios gestionan un total de 1,3 millones de ovejas (Pozo y Agudo, 2013). El grupo OVISO ha experimentado un enorme crecimiento desde finales de la década de 1990 hasta la actualidad, no solo en tamaño (se ha pasado de 3 cooperativas a 17) sino también en lo que respecta a la introducción de mejoras en la cadena de valor, donde se ha reforzado la formación de los ganaderos, la inversión en infraestructuras productivas y la creación de centros de tipificación y cebo.

Una de las estrategias entendidas por la cooperativa como clave de futuro ha sido la búsqueda de colaboraciones y acuerdos con otras cooperativas del sector para alcanzar nuevos mercados. Ejemplo de ello es Ovispain, que es un consorcio para la exportación, constituido por Oviso, Grupo Pastores y Cordesur. Esta colaboración ha permitido que se haya pasado de exportar 4.108 canales en el año 2005 (solo Oviso) a más de 70.000 canales en el año 2012 (Ovispain). Con esta colaboración se pretende buscar alternativas en las épocas de bajo consumo nacional.

Las tres cooperativas se ubican en la zona oriental de la provincia de Badajoz, en áreas de características edafoclimaticas muy similares, con suelos muy pobres y escasa producción de pastos. Los cooperativistas acceden a los servicios de la cooperativa de segundo grado en las mismas condiciones. Aunque el asesoramiento técnico a nivel de explotación sea similar, el personal técnico que da asistencia depende de la cooperativa de primer grado. En cuanto a las razas mayoritariamente explotadas, son fundamentalmente de base racial Merina con cruce a partir de machos mejorantes (Ille de France y Merino Precoz fundamentalmente).

\section{OBTENCIÓN DE LA INFORMACIÓN}

A partir de la información primaria suministrada por las cooperativas, se elaboró una base de datos depurada que se completó con fuentes secundarias y trabajo de campo. Esta base de datos constituye el punto de partida para la elaboración de los indicadores, así como de fuente de información dentro del proyecto de investigación.

Tabla III. Indicadores productivos. Media, error estándar y nivel de significación (ANOVA) en las explotaciones en función de la cooperativa de pertenencia (Productive indexes. mean, standard error and significance level (ANOVA) in the farms according to cooperative membership).

\begin{tabular}{|c|c|c|c|c|c|c|}
\hline Indicadores & Coop 1 & Coop 2 & Coop 3 & Total muestra & ESM & Sig. \\
\hline $\begin{array}{l}\text { Productividad comercial } \\
\text { (cordero entregado/oveja) }\end{array}$ & 0,87 & 0,94 & 0,88 & 0,89 & 0,02 & NS \\
\hline $\begin{array}{l}\text { Precio medio cordero } \\
(€ / \text { cordero entregado) }\end{array}$ & $66,58^{\mathrm{ab}}$ & $65,56^{\mathrm{a}}$ & $67,16^{\mathrm{b}}$ & 66,59 & 0,2 & * \\
\hline $\begin{array}{l}\text { Precio medio por } \mathrm{kg} \text { de } \\
\text { peso vivo de cordero } \\
\text { entregado } \\
(€ / \mathrm{kg})\end{array}$ & 3,24 & 3,24 & 3,19 & 3,22 & 0,01 & NS \\
\hline $\begin{array}{l}\text { Precio medio de oveja } \\
\text { desvieje } \\
\text { (€/oveja) }\end{array}$ & $24,88^{a}$ & $25,74^{\mathrm{a}}$ & $29,90^{\mathrm{b}}$ & 26,87 & 0,75 & * \\
\hline $\begin{array}{l}\text { Kg de lana por oveja adulta } \\
\text { (kg/oveja) }\end{array}$ & $2,88^{a}$ & $2,27^{b}$ & $4,80^{c}$ & 3,53 & 0,13 & $* *$ \\
\hline $\begin{array}{l}\text { Precio medio de la lana } \\
(€ / \mathrm{kg})\end{array}$ & $1,37^{a}$ & $1,37^{a}$ & $0,77^{\mathrm{b}}$ & 1,10 & 0,03 & $* *$ \\
\hline
\end{tabular}


La información aportada por Oviso se circunscribió fundamentalmente a datos técnicos de las explotaciones del año 2012, siendo necesario contactar directamente con cada cooperativa asociada para obtener información más concreta de la actividad de los ganaderos. Se ha podido comprobar que la disponibilidad y uso de la información almacenada por parte de las cooperativas de primer grado es limitada, requiriendo de una intensa colaboración por parte de los técnicos para la elaboración final de la base de datos. La figura 1 muestra el proceso metodológico seguido en este trabajo.

\section{VARIABLES DE ESTUDIO Y GENERACIÓN DE INDICADORES}

La caracterización de explotaciones es frecuente a la hora de analizar los sistemas de producción animal (Gibon, et al., 1999). En la bibliografía es posible encontrar trabajos basados generalmente en características estructurales, productivas y económicas de las explotaciones como los de Castel et al. (2003), Milán et al. (2003), Maseda et al. (2004), Riedel et al. (2005), Usai et al. (2006), y más recientemente, con la incorporación de criterios de sostenibilidad y la prestación de servicios ecosistémicos de las explotaciones, como son los trabajos de Gaspar et al. (2009) y Ripoll-Bosch et al. (2012). Estos estudios fueron tenidos en cuenta en el diseño de indicadores técnico-económicos que permitiesen llevar a cabo el análisis en profundidad de las características productivas básicas de las explotaciones.

\section{TRATAMIENTO Y ANÁLISIS DE INFORMACIÓN}

El tratamiento y análisis estadístico de la información contenida en la base de datos definitiva se realizó con el paquete estadístico SPSS, v.22, debido a su potencia de análisis, capacidad de generación de informes y facilidad para importar datos del Excel.

Se calcularon estadísticos descriptivos de las distintas variables analizadas. Además, se aplicaron test paramétricos (Análisis de la varianza) para determinar los contrastes de significación entre las distintas cooperativas.

\section{RESULTADOS Y DISCUSIÓN}

\section{Características de las eXPLOTACIONES ESTUdIADAS}

La tabla I contiene la información relativa a los a los indicadores estructurales de las explotaciones así como información de carácter cualitativo relevante. Estos parámetros permiten identificar algunas diferencias estructurales de base en cuanto a la dimensión de las explotaciones estudiadas, tanto en superficie disponible como en censos ovinos, en la titularidad de la tierra y en los usos del suelo para fines agrícolas. Además también se reflejan diferencias en cuanto a las razas predominantes en las cooperativas así como en algunas prácticas de manejo ganadero.

\section{ANÁLISIS DE LOS ÍNDICES TÉCNICOS DE LAS COOPERATIVAS}

En este primer apartado se aborda el análisis de la gestión de la reproducción y de la alimentación de los rebaños a partir de los índices técnicos más relevantes. En la tabla II aparecen los valores medios de estos índices para cada una de las cooperativas analizadas.

Uno de los indicadores con gran relevancia del rendimiento ganadero ovino es la tasa de reposición propia. En este caso los valores que presentan las tres cooperativas parecen ajustarse a los requerimientos habituales de las explotaciones ovinas extensivas, alrededor del 15\% de reposición, la cual cubre por término general las bajas y el desvieje anual de animales. No obstante, se observa que la cooperativa 2 tiene unos valores sensiblemente superiores a los medios. El valor del porcentaje de reposición de ovejas puede tomarse como referencia a la hora de decidir si la explotación necesita una inspección más detallada, ya que aquellas explotaciones con tasas por debajo del 13\% o por encima de $20 \%$ se salen de los parámetros aconsejables.

Otra de las problemáticas que en ocasiones se puede encontrar en una explotación de ovino de carne en extensivo es la merma de la capacidad reproductiva de las ovejas debida a una escasez de sementales o machos para la cubrición en la explotación (ratio ovejas reproductoras/semental). Como se puede observar en la tabla II, existen diferencias significativas entre las

Tabla IV. Indicadores económicos. Media, error estándar y nivel de significación (ANOVA) en las explotaciones en función de la cooperativa de pertenencia (Economic indexes. mean, standard error and significance level (ANOVA) in the farms according to cooperative membership).

\begin{tabular}{|c|c|c|c|c|c|c|}
\hline Indicadores & Coop 1 & Coop 2 & Coop 3 & Total muestra & $\mathrm{ESM}^{1}$ & Sig. \\
\hline Total gastos de mano de obra por oveja (€/oveja) & $5,99 a$ & $19,35 b$ & $14,20 b$ & 12,92 & 1,01 & ** \\
\hline Gastos sanitarios totales por oveja (€/oveja) & $2,71 \mathrm{a}$ & $4,30 \mathrm{~b}$ & $3,37 a b$ & 3,39 & 0,21 & * \\
\hline Gasto bruto total por oveja (€/oveja) & 59,92 & 59,7 & 56,55 & 58,35 & 2,27 & NS \\
\hline Gastos contratación agrícola/gastos brutos & $0,34 a$ & $0,04 b$ & $0,28 a$ & 0,24 & 0,02 & ** \\
\hline Gastos de alimentación/gastos brutos & $0,53 a b$ & $0,61 \mathrm{a}$ & $0,47 b$ & 0,52 & 0,01 & * \\
\hline Gastos de mano de obra/ gastos brutos & $0,13 a$ & $0,34 b$ & $0,29 b$ & 0,25 & 0,02 & ** \\
\hline Ingresos por subvenciones por oveja $(€)$ & 38,53 & 49,04 & 48,35 & 45,38 & 2,13 & NS \\
\hline Ingreso bruto total por oveja (€/oveja) & 102,68 & 117,21 & 110,11 & 109,54 & 2,98 & NS \\
\hline Margen bruto total por oveja (€/oveja) & $42,76 a$ & $57,50 b$ & $53,56 a b$ & 51,19 & 2,96 & * \\
\hline
\end{tabular}

${ }^{*} p<0,05 y{ }^{* *} p<0,01 ;{ }^{1} E S M$ : Error estándar de muestreo.

Diferentes subíndices en la misma fila señalan diferencias significativas para ${ }^{*} p<0,05 y{ }^{* *}<0,01$. 


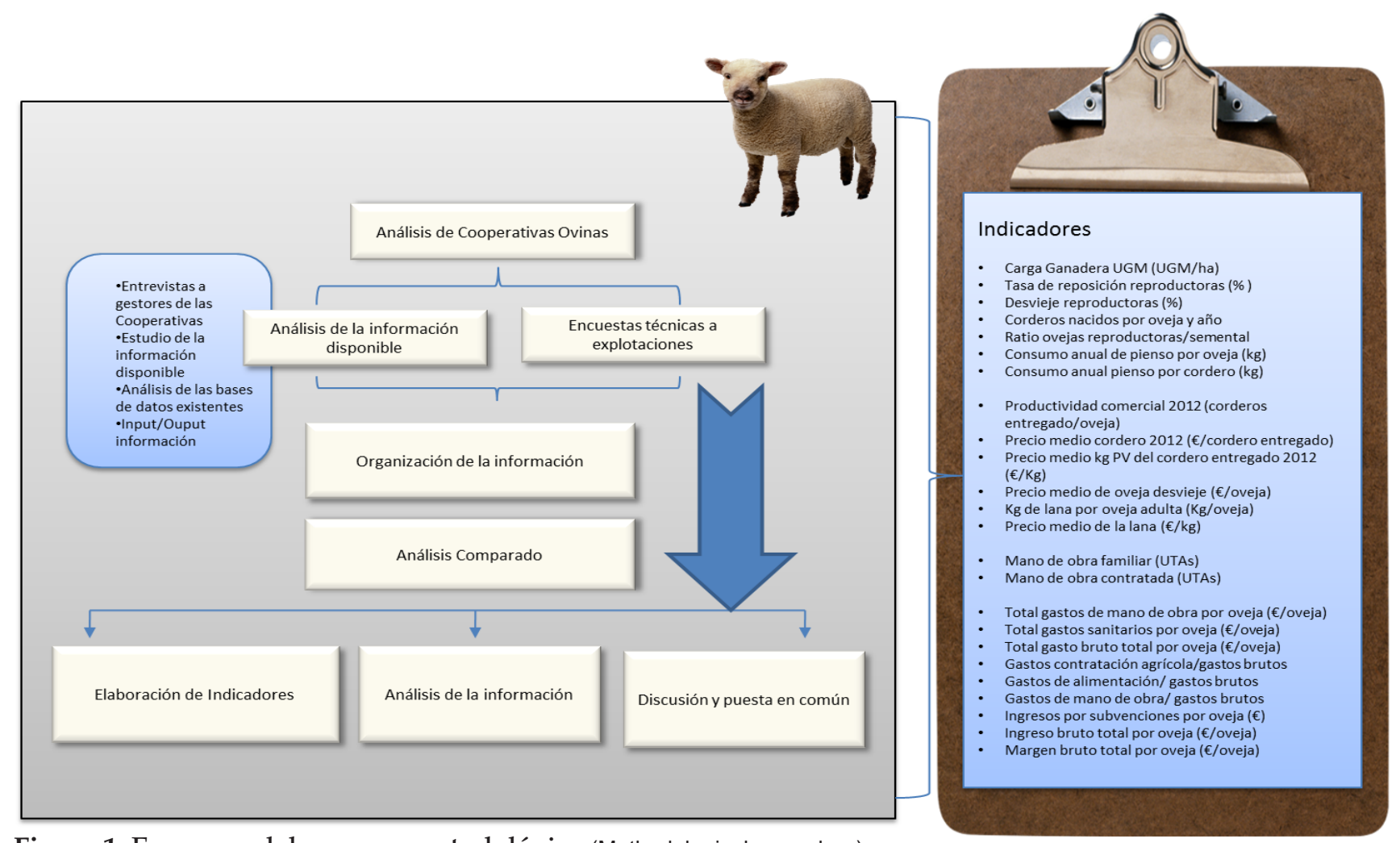

Figura 1. Esquema del proceso metodológico (Methodological procedure).

tres cooperativas a este respecto, siendo la Cooperativa 2 la que tiene este ratio menor, con 26,33 ovejas por semental.

A la vista de estos resultados, la cooperativa 2 muestra una mejor gestión de la reproducción obteniendo una mayor productividad de corderos $(1,22$ nacidos/oveja/año). Sin embargo, al comparar todas las cooperativas con indicadores de gestión reproductiva de otros sistemas extensivos ovinos se observa que dichos indicadores son ligeramente superiores a los observados por Gaspar et al. (2007) y Milán et al. (2003) por lo que se deriva que las explotaciones en el entorno cooperativo son más eficientes en estos aspectos gracias a la asistencia técnica y al asesoramiento especifico en temas reproductivos.

Además de los indicadores expuestos anteriormente, hay que destacar el parámetro carga ganadera. Estas cargas ganaderas son elevadas para el tipo de explotación de ganado ovino en extensivo donde los valores más recomendados están en torno a las 0,3 UGM / ha. Esa mayor intensidad en el uso de los recursos pastables es habitual en los sistemas ovinos de zonas semiáridas y se viene observando desde hace ya varias décadas, ya que ha sido la forma más habitual de maximizar la percepción de subvenciones durante los periodos en que éstas estaban vinculadas al número de cabezas.

En relación a la gestión de la alimentación del ganado, cabe señalar que las variaciones que se puedan encontrar están estrechamente relacionadas con el parámetro de carga ganadera anteriormente analizado. En la figura 2 se observa el consumo medio de pienso de reproductores por oveja, donde destaca que las cooperativas con menor carga ganadera tienen menores consumos ya que tienen más recursos del medio a disposición de cada animal. La figura 3 muestra el consumo medio de pienso por cordero de cebo donde se puede observar que apenas hay diferencias entre las cooperativas. Este hecho es esperable porque los sistemas de cebo son muy similares en condiciones intensivas donde la cooperativa de segundo grado estandariza los pesos de salida de los corderos.

\section{GESTIÓN DE LA MANO DE OBRA}

Las figuras 4 y 5 muestran la estructura de la mano de obra por explotación, así como su productividad. Así, se aprecian diferencias en lo relativo al tipo de mano de obra utilizada en cada cooperativa, observándose que en las explotaciones de las cooperativas 1 y 3 la mano de obra es fundamentalmente familiar, mientras que en las explotaciones de la cooperativa 2 se utiliza más la mano de obra contratada. Este dato está relacionado con el mayor número de animales que presentan las explotaciones de esta cooperativa, que hace necesario contar con personal externo. En relación a la productividad de la mano de obra, destaca que en general son valores muy superiores a los encontrados en otros estudios de sistemas ovinos de carne cooperativos (Pardos et al., 2008).

\section{ANÁLISIS DE LOS INDICADORES PRODUCTIVOS}

La tabla III refleja los resultados obtenidos sobre la producción y venta de corderos.

En relación a los resultados obtenidos sobre la productividad comercial de las tres cooperativas analizadas, se observa que el precio medio del cordero para cada cooperativa se encuentra en rangos muy cercanos. 
Aunque se las diferencias son poco significativas (son significativas entre la 2 y la 3), la cooperativa 2 es la que de media ingresa menos por cordero entregado.

Estos indicadores de venta de corderos, aunque interesantes para su análisis y comparación entre las tres cooperativas, también sirven para estudiar una de las cuestiones que más interesan como gestores en una explotación: la productividad comercial por oveja.

La Productividad Comercial anual por oveja es una variable normalizada que indica el número total de corderos entregados a la cooperativa por oveja reproductora a lo largo de un año. Este es un indicador de gran importancia para la cooperativa, ya que expresa la relación de producción entre asociado y empresa. De nuevo la cooperativa 2 es la que presenta el valor medio más alto $(0,94)$ lo que indica que es la cooperativa cuyos socios entregan un mayor número de corderos.

Si analizamos de una forma práctica este indicador, es decir, desde la perspectiva de la cooperativa, se podría evaluar el comportamiento del asociado en relación a la misma. En este análisis solo se incluirían los ganaderos que entreguen por debajo de una tasa de entrega que se considere como la deseable para ese tipo de explotaciones, debiéndose estudiar los motivos que subyacen en tasas inferiores para así poder establecer mecanismos para incrementar dichos niveles.

\section{BALANCE DE GASTOS E INGRESOS DE LAS EXPLOTACIONES}

La tabla IV muestra los resultados de los principales indicadores económicos obtenidos para cada cooperativa.

En la tabla IV se aprecian diferencias significativas entre las cooperativas, algunas de las cuales, como el gasto en mano de obra por oveja o los gastos de contratación agrícola/gasto brutos, están claramente relacionados con el porcentaje de mano de obra familiar que ya se ha explicado anteriormente.

Son también destacables los indicadores más puramente económicos, como el ingreso bruto total por oveja o el margen bruto total por oveja, en los que la cooperativa 2 destaca con respecto a las otras dos. Una explicación a este comportamiento puede estar en la estructura de las explotaciones de la cooperativa 2 , que eran las que tenían mayor número de ovejas y carga ganadera, con raza mayoritariamente merina y que presentaban la mayor tasa de corderos nacidos, así como la mayor productividad comercial (corderos entregados por oveja). Esta relación entre la rentabilidad y los indicadores de productividad se puede apreciar también en trabajos como el de Pardos et al. (2008), aunque en dicho estudio los datos no son directamente comparables al tratarse de razas con un comportamiento muy diferente a las que se explotan en esta zona.

La mayor carga ganadera no parece ser, no obstante, la principal explicación de la rentabilidad de las explotaciones, a la luz de trabajos como el de Gaspar et al. (2008). En dicho trabajo, aunque la tasa de rentabilidad de las dehesas analizadas se incrementaba con la carga ganadera, el factor que parecía tener más relevancia era finalmente la explotación

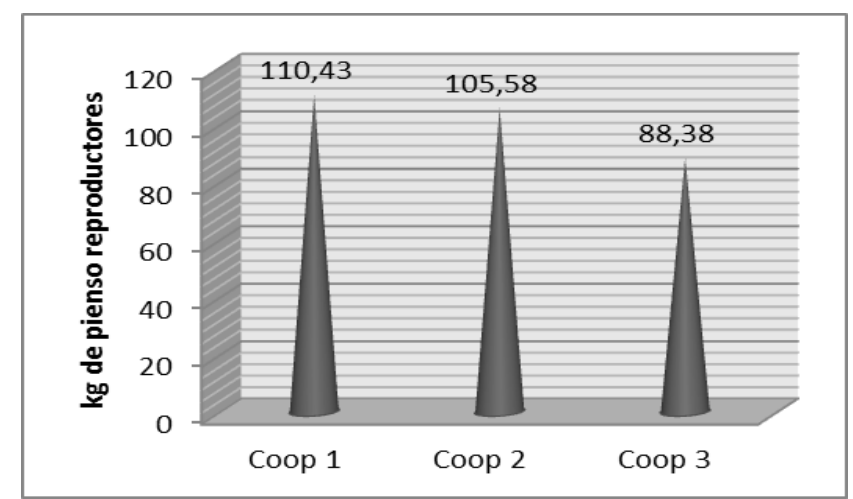

Figura 2. Consumo medio anual de pienso por oveja (Average yearly consumption of concentrate per sheep).

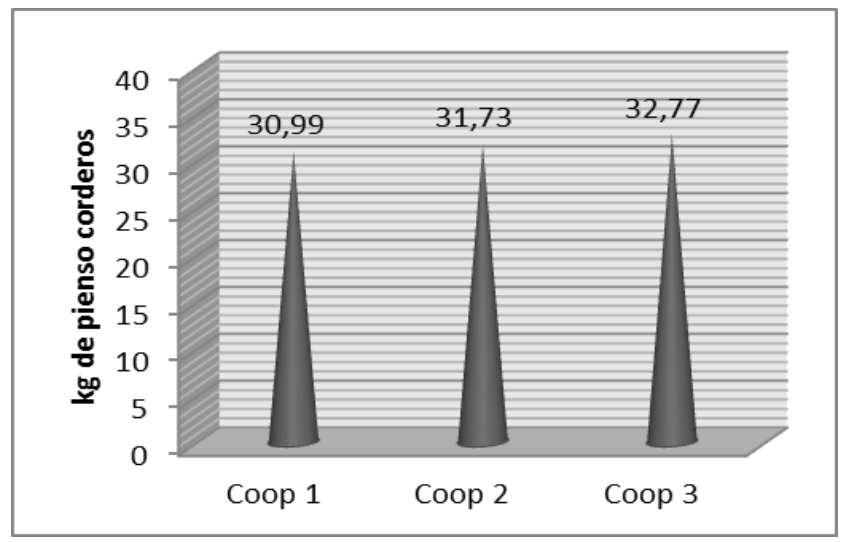

Figura 3. Consumo medio anual de pienso por cordero (Average yearly consumption of concentrate per lamb).

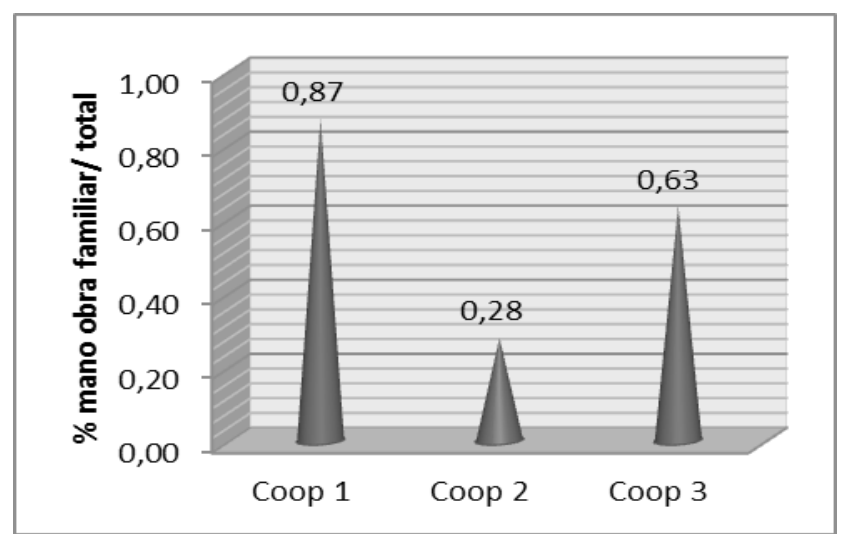

Figura 4. Relación entre la mano de obra familiar y la mano de obra externa (Relationship between family and external labour).

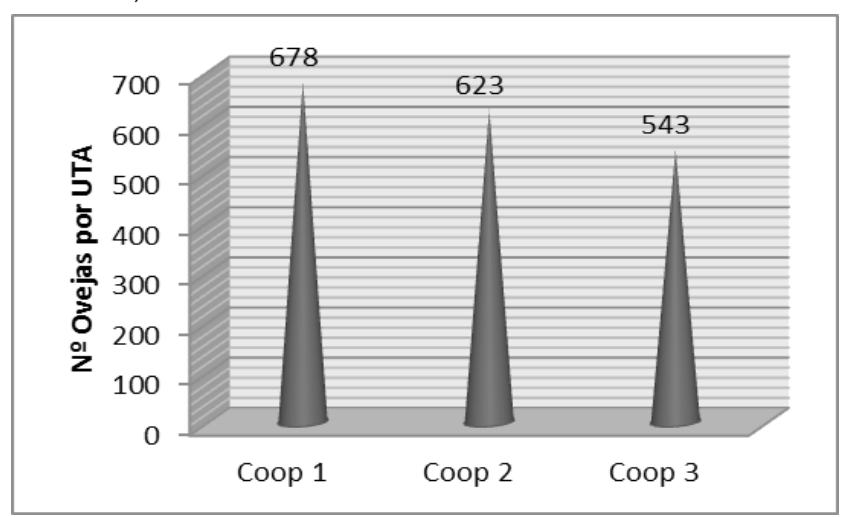

Figura 5. Productividad de la mano de obra (Labour productivity). 
de diversas razas como forma de aprovechar más eficientemente los recursos del medio.

\section{CONCLUSIONES}

El estudio comparado de las explotaciones ovinas pertenecientes a tres cooperativas, integradas en una única entidad de segundo grado, ha permitido identificar aspectos homogéneos tales como el precio medio por $\mathrm{kg}$ de peso vivo obtenido por los ganaderos en cada cordero entregado. Este hecho era previsible ya que la comercialización de los corderos se hace de forma conjunta. Sin embargo también se han encontrado aspectos diferenciados entre las cooperativas, como los relacionados con el manejo reproductivo (tasa de reposición, ratio de reproductoras por semental y gastos veterinarios) y que podrian estar ligados a la diferente aplicación del asesoramiento recibido.

Así, y aunque en todas las cooperativas analizadas las explotaciones han mostrado cargas ganaderas superiores a las recomendadas para entornos semiáridos, aquellas con menores cargas lo han reflejado en su menor dependencia de los piensos comprados, ya que pueden hacer un mejor aprovechamiento de los recursos del sistema.

Se han apreciado interesantes diferencias con respecto a la productividad, ya que la Cooperativa 2 presenta valores sustancialmente más elevados. Las diferencias en un aspecto tan importante para la cooperativa pueden mostrar un aspecto en el que se podrían llevar a cabo mejoras relevantes.

Finalmente, y con respecto a los indicadores económicos, la cooperativa 2 destaca de nuevo. Las posibles explicaciones a estos datos podrían venir por la propia estructura de las explotaciones socias (son las de mayor número de ovejas y carga ganadera, con raza mayoritariamente merina y la mayor tasa de corderos nacidos), aunque de la comparación con otros estudios similares se podría pensar más bien en una relación entre la rentabilidad y los indicadores de productividad, aspecto este que tendría que ser objeto de estudios más amplios para poder acometer las medidas de mejora pertinentes.

El estudio diferenciado de entidades en un mismo entorno cooperativo empresarial podría servir tanto como para establecer estrategias de benchmarking (las mejores explotaciones se convertirían en granjas modelo para el resto de los cooperativistas), como para corregir deficiencias, al permitir detectar valores anormales o comportamientos poco eficientes.

\section{AGRADECIMIENTOS}

Los autores agradecen el apoyo y la financiación proporcionados por la Junta de Extremadura a través del Proyecto de Investigación PCJ100901, cofinanciado por los Fondos Europeos FEDER. También quieren expresar su agradecimiento al equipo técnico de Oviso y sus cooperativas socias, por su valiosa aportación en la toma de datos.

\section{BIBLIOGRAFÍA}

Bernués, A.; Ruiz, R.; Olaizola, A.; Villalba, D. and Casasús, I. 2011. Sustainability of pasture-based livestock farming systems in the European Mediterranean context: Synergies and trade-offs. LivestSci, 139: 44-57.

Castel, J.M.; Mena, Y.; Delgado-Pertinez, M.; Camúñez, J.; Basulto, J.; Caravaca, F.; Guzmán-Guerrero, J.L and Alcalde, M.J. 2003. Characterization of semi-extensive goat production systems in southern Spain. Small Ruminant Res, 47: 133-143.

De Rancourt, M.; Fois, N.; Lavín, M.P.; Tchakérian, E. and Vallerand, F. 2006. Mediterranean sheep and goats production: An uncertain future. Small Ruminant Res, 62: 167-179.

Escribano, M.; Rodríguez, A.; Mesías, F.J. and Pulido, F., 2001. Relationship between the farm size and stocking rate in extensive sheep systems. Livestock Research for Rural Development, 13: 1-5.

Gaspar, P.; Escribano, A.; Mesías, F.J.; Ledesma, A.R. and Pulido, F. 2008. Sheep farms in the Spanish rangelands (dehesas): Typologies according to livestock management and economic indicators. Small Ruminant Res, 74: 52-63.

Gaspar, P.; Mesías, F.J.; Escribano, M.; Ledesma, A.R.D. and Pulido, F. 2007. Economic and management characterization of dehesa farms: Implications for their sustainability. Agroforest Syst, 71: 151-162.

Gaspar, P.; Mesias, F.J.; Escribano, M. and Pulido, F., 2009. Assessing the technical efficiency of extensive livestock farming systems in Extremadura, Spain. Livest Sci, 121: 7-14.

Gibon, A.; Sibbald, A.R.; Flamant, J.C.; Lhoste, P.; Revilla, R.; Rubino, R. and Sørensen J.T. 1999. Livestock farming systems research in Europe and its potential contribution for managing towards sustainability in livestock farming. Livest Prod Sci, 61: 121-137.

Horrillo, A.; Escribano, M.; Mesias, F.J.; Elghannam, A. and Gaspar, P. 2016. Is there a future for organic production in high ecological value ecosystems? Agr Syst, 143: 114-125.

MAGRAMA. 2014. Caracterización del sector ovino y caprino en España. Año 2014. Ministerio de Agricultura, Alimentación y Medio Ambiente. Dirección General de Producciones y Mercados Agrarios. Subdirección General de Productos Ganaderos, 25 pp.

Maseda, F.; Díaz, F. and Álvarez, C. 2004. Family dairy farms in Galicia (N.W. Spain): Classification by some family and land factors relevant to quality of life. Biosystems Engineering, 87(4): 509-521.

Mena, Y.; Ruiz-Mirazo, J.; Ruiz, F.A. and Castel, J.M., 2016. Characterization and typification of small ruminant farms providing fuelbreak grazing services for wildfire prevention in Andalusia (Spain). Sci Total Environ, 544: 211-219.

Milán, M.J.; Arnalte, E. and Caja, G. 2003. Economic profitability and typology of Ripollesa breed sheep farms in Spain. Small Ruminant Res, 49: 97-105.

Pardos, L.; Rubio, M.M. and Fantova, E. 2008. The diversity of sheep production systems in Aragón (Spain): characterisation and typification of meat sheep farms. Span J Agric Res, 6: 497-507.

Pozo, J.C. y Agudo, B. 2013. El ovino de carne cooperativo en Extremadura. En: La agricultura y ganadería extremeñas, informe 2012. Ed. Facultad de Ciencias Económicas y Empresariales, Escuela de Ingenierías Agrarias y Caja de Badajoz. España, pp. 121-133.

Riedel, J.L.; Casaús, I.; García, A.; Sanz, A.; Revilla, R. y Bernués, A. 2005. La ganadería ovina en el Parque de la Sierra y Cañones de Guara. I- Intensificación reproductiva y manejo del pastoreo. ITEA, 26 (I): 189-191.

Ripoll-Bosch, R.; Diez-Unquera, B.; Ruiz, R.; Villalba, D.; Molina, E.; Joy, M.; Olaizola, A. and Bernues, A. 2012. An integrated sustainability assessment of Mediterranean sheep farms with different degrees of intensification. Agr Syst, 105: 46-56.

Ruiz, M.C.; García, E. y Hernández, M.J. 2014. Cómo responden a la crisis económica actual las sociedades cooperativas agrarias. El caso de las almazaras cooperativas andaluzas. REVESCO: revista de estudios cooperativos, 113: 120-149.

Usai, M.G.; Casu, S.; Molle, G.; Decandia, M.; Lidgios, S. and Carta, A. 2006. Using cluster analysis to characterize the goat farming system in Sardinia. Livest Sci, 104: 63-76. 